\title{
Evolving from Principles of GV Black
}

\section{A Parameswaran}

\begin{abstract}
The Father of Modern Dentistry, Greene Vardiman Black, had played a vital role in elevating the dental profession to an autonomous status. Modern dentistry has continuously evolved from the time of GV Black. Now, there has been a paradigm shift in concept from 'Extension for prevention' to 'Restriction with conviction'. 'Medical model' of caries management has been recognized as the most logical approach for success. The prediction of GV Black that 'The professional person has no right to be other than a continuous student' has become a reality. It is the duty of every conservative dentist to appreciate and adopt the changes and this will be the real tribute paid to the pioneers of the profession.
\end{abstract}

Keywords: Evolving principles, GV black, Modern dentistry.

How to cite this article: Parameswaran A. Evolving from Principles of GV Black. J Oper Dent Endod 2016;1(1):3-6.

Source of support: Nil

Conflict of interest: None

\section{INTRODUCTION}

Greene Vardiman Black (1836-1915)—My appreciation for that 'Genius' of dental education recalled my days of dentistry both as student and teacher and I take this opportunity to dedicate this article following his 100th year of the demise on August 31st 1915.

Black, the man as detailed by Schewe, 'was an Alumnus and Faculty of Missouri's Dental College and remains an unforgettable character in this field. During his teens, he went to live with his elder brother Mr Thomas Black, a physician in Illinois. He met a dentist Mr JC Spear at Mount Sterling where he decided to support his mentor and guide in dental practice as an apprentice. He relocated later to Winchester after developing the necessary skill and became the first dentist. He was a success in his profession and later enrolled in the union army during the civil war. Black, was enlisted as a member when the Missouri's State Dental Association met during June 1866. He became a faculty in Missouri's Dental College (1870-1881) and was awarded DDS degree. Though he

Former Principal

Tamil Nadu Government Dental College and Hospital, Chennai Tamil Nadu, India

Corresponding Author: A Parameswaran, Former Principal Tamil Nadu Government Dental College and Hospital, Chennai Tamil Nadu, India, e-mail: param1809@gmail.com lacked formal education he was considered, a 'prodigy in dentistry' and was recognized to receive several honorary degrees like DDS, MD, ScD, LLD.

'The Father of Modern Dentistry' GV Black had played a vital role in elevating the dental profession to an autonomous status. He mastered many languages and had many text books to his credit. His publication in 1891 in 'Dental Cosmos' on management of enamel margin became a unique contribution in which he framed the popular phrase, 'Extension for prevention' which became one of the salient steps in principles of cavity preparation. His experiment in using dental amalgam as a filling material became immortal by his research and he established the fact to the world that scientifically prepared silver amalgam when used could be a successful filling material. He developed special hand cutting instruments for cavity preparation and also for condensation of silver amalgam. He conceptualized a standard formula for making silver alloy which was absorbed by manufacturers for commercial production. Between the year 1941 and 1945, the army dentists inserted nearly one crore amalgam restorations to the service personals!

'In spite of being the material most used practically, amalgam has also been the material most abused verbally.'

It all started from GV Black, whose primary contribution to dentistry, was the classification of carious lesions in relation to the presence of caries on teeth surface. They were classified as class I to V and some were compounded by combinations of surfaces. In his classification, he did not specify the decay present on the cusps of posterior and tips of anterior teeth. They were added as class VI later. This method of identifying dental caries is still in vogue and is followed by dental students and practitioners for better understanding of the presence of decay and its further management. The management of decay changed according to the area affected and it helped to decide the material of choice for restoration.

Modern classification of dental caries according to Sturdevant ${ }^{2}$ introduced numerous changes. Primary caries were classified according to location as pit and fissure, smooth surface and root caries. According to direction of spread, they were classified as forward and backward. According to the extent of decay, they were known as insipient or minimal caries. According to the rate of spread, they could be acute (rampant) 
caries or chronic and slow caries. According to depth of penetration, they were known as enamel caries or dentinal caries. Secondary caries were seen as reinfection under the existing restorations.

There is a clinical significance in this newer classification. Minimal caries which are present in pits and fissures are managed by sealing the pits and fissures to make them resistant to caries. Root surface caries are called senile caries and are mostly seen in older age group patients due to the recession of the gingival attachment toward the root or due to restricted salivary flow or poor oral hygiene. Root caries progress faster and should be managed holistically by addressing to the situation. If the caries activity is slow, it might get arrested in progress due to various reasons, such as improved oral hygiene and increase of fluoride in saliva. Early detection of caries could be done by noticing the presence of white spots in enamel which are due to demineralization by acid action. Judicious methods of management are recommended in those cases.

Black, brought out a list of principles of cavity preparation, the most important being the outline form. The cavity should include all the caries affected areas and the final preparation must be preconceived by the dentist prior to restoration. He had included 'removal of remaining decay' and placement of margins in selfcleansing areas and suggested the margins of the cavity should not face the occlusal forces of the opposing tooth to avoid fracture. Black, suggested the depth of $0.5 \mathrm{~mm}$ below the dentinoenamal (DE) junction for an ideal cavity preparation. In proximal cavity preparations he even suggested that the gingival margins should be apical to the crest of the free gingiva to avoid secondary caries.

Black's cavity preparation had gone through a major change over the years. His concept of 'Extension for prevention' during the preparation of proximal cavity has lost its significance due to the change of concept that aims toward conserving the tooth structure. Bronner ${ }^{5}$ advocated conservative cavities for class II amalgam by using proximal locks instead of extensive occlusal steps. He also advocated rounded internal line angles and less prominent point angles to avoid fracture of teeth. To facilitate better retention of fillings the walls were prepared using pear-shaped burs and proximal portion of the cavity was prepared conservatively by using smaller burs. According to Bronner, the bur when used, to prepare a class II cavity penetrated the dentin just inside the enamel wall to form a narrow occlusal opening and when swayed to create broader base toward gingiva beyond the contact area, resulted in a slot preparation. The enamel wall thus separated could be removed by a small bur or using a hand-cutting instrument. Further gingival preparation could be finished with a pear-shaped bur to form rounded line angles and gingival marginal trimmer on the enamel margin.

Occlusal retention locks were prepared in the sound dentin by a tiny fissure bur and finished with a pearshaped bur to increase retention. ${ }^{3}$ The axiogingival line angle could be defined and the axiopulpal line angle was left rounded. The isthmus was minimized to one-fourth of the intercuspal distance, whereas GV Black recommended a broader isthmus which was one-third of the intercuspal distance. Important principle of conservative approach was that each part of the cavity design had to be made self-retentive for the restoration. Some of the Black's principles for cavity design for tooth-colored preparations were modified by several operators to improve esthetics. Before the introduction of adhesive dental restorative materials, esthetics was improved when restoring anterior teeth by selective modification of the cavity preparation. Woodbury $\mathrm{CE}^{4}$ suggested labial enamel margin of the preparation to be in harmony with the labial surface enamel. Major breakthrough came with the invention of composites which were retained by means of acid etching and bonding. The retention depends on the geometric component produced when the resin penetrates into and locks with the micro porosities of the etched tooth surface.

Fusion achieved by certain chemical material to enamel and dentin created a breakthrough in cavity designing. Introduction of polycarboxylates with aluminosilicates (glass-ionomer) had both the advantage of better union to dental tissues and pulp friendly. Optimum attachment to the cavity wall was also obtained with the use of conditioners. Being adhesive to tooth enamel and dentin, mechanical retentive aids were not needed in cavity design. These materials possessed physiochemical adhesive ability to the part of the tooth structure. Addition of fluoride in its composition with the ability to leach helped to prevent caries activity in and around the filling. Glass ionomer had also found a place to be used as a liner under composite restorations, as a 'bilayer' restoration. This would inturn protect the pulp under direct composite restorations.

GV Black had predicted 'The Professional person has no right to be other than a continuous student.'

He had a vision, that one day prevention of dental caries will change the principles of cavity preparation proposed by him. This had come true over continuous research, as the conservative cavity preparation has become the norm of the day with the introduction of adhesive materials.

'Minimum intervention', by Mount GJ, ${ }^{6}$ was a turning point in cavity designs which were under constant review and change. Caries being recognized as a bacterial 
disease the entire treatment procedure of dental caries got modified as a 'medical model'. Medical management of caries risk had become popular in place of surgical management by cavity preparation. Understanding and controlling the risk factors are used to reduce the pathological factors and to increase the preventive factors. Additionally, there are methods to remineralize the loss to avoid any operative intervention.

Only advanced caries lesion is surgically managed to eliminate the decay and its cause, whereas the smaller caries affected areas were managed differently and it differed from that suggested by GV Black. For following minimum intervention, suitable training was given to practitioners. Here, I remember our earlier private practitioners who revolutionized by doing 'dot' filling with a minimal sized drill and filled with silver amalgam when treating pit caries on enamel surface. They had practiced 'minimum intervention' in caries management much ahead of time and technology. The prime aim was to prevent and heal the dental disease by reducing the chance of an extensive intervention.

'Restriction with conviction instead of extension for prevention' - Charles Wakefield.

It was clear that no restorative material was recognized as permanent, as it may result in further damage to the material or the tooth structure during passage of time and use. Replacement with suitable restoration to simulate the original tooth anatomy was difficult and may lead to changes in occlusal harmony. Black's cavity design required geometric precision with sharp line angles and flat floors which may lead to excess removal of tooth structure. Whereas minimum intervention suggested remineralization of enamel surface which had not developed a cavity and remineralization of the affected dentin on the floor of the cavity which prevented pulpal irritation. Remineralization could be achieved on enamel with application of fluoride. Glass ionomer foundation for a restoration would also help in sealing the margin from microleakage.

\section{CLASSIFICATION OF CAVITY DESIGN BY GRAHAM MOUNT}

\section{Lesion Site}

- Site 1: Pit and fissures of posteriors.

- Site 2: Contact areas of anterior and posterior teeth.

- Site 3: Cervical areas coronal to gingival margin and areas including exposed root surface.

\section{Size of the Lesion}

- Size 0: Initial lesion at any site

- Size 1: Lesion with small cavitation needing surgical intervention
- Size 2: Moderate size of the cavity with sufficient sound tooth structure which would accept occlusal load.

- Size 3: Cavity needs enlargement and modification for protecting the remaining part of the tooth from occlusal load.

- Size 4: Extensive cavity with loss of cusp of anterior or posterior teeth.

Graham Mount suggested the use of small size burs for minimal surgical management and had given the other options using laser cutting of hard tissues and air-abrasive methods.

'Modern methods for removal of diseased tooth structure should be used primarily to enhance patient comfort but at the same time undue loss of sound tooth structure must be avoided. While precise geometric cavity designs are no longer to be contemplated, any sacrifice of sound tooth structure is to be deprecated. Good visibility and a high level of tactile sense are useful tools in limiting the extent of surgical removal of tooth structure'-Mount G.

Atraumatic restorative treatment $(\mathrm{ART})^{7}$ developed as an effective minimal intervention approach to manage dental caries by Frencken J (University of Nijmegen) and Holmgren C (University of Hong Kong), and was approved by WHO. The innovative treatment approach of ART was originally developed to manage dental carries in larger population without relying on expensive dental equipment. It was based on excavation of caries with hand instruments followed by restoration of an adhesive filling material by compressing with a gloved finger. Though this would appear to be a simplistic and a retrograde step in the face of ever increasing sophistication in dentistry this was practiced in massive dental camps conducted in underdeveloped countries where electricity is a luxury, and this could be considered synonymous to the service of 'bare-foot' doctors.

GV Black performed extensive research and devised specific hand cutting instruments to perform detailed geometrically accurate cavity preparation. Recent text books of operative dentistry mention only certain selected hand-cutting instruments, like gingival marginal trimmers which are still accepted and used for the preparation of gingival enamel margin to the recommended bevel, and hand excavators to remove soft decay without damaging the dental pulp. Present day students of dentistry read about hand-cutting instruments merely as an academic exercise.

This early scientific dentist GV Black's systematic approach to understand dental caries and its management had undergone phenomenal changes with the advent of new generation of materials in the management of dental 
caries. Future of dentistry would be guided by technology and would create new methodology for handling caries teeth.

This could be a Eulogy for the evolution of operative dentistry over the past few decades with a metamorphosis from 'Drill and Fill' to 'Clean and Fill'.

'Since the time, it has required many years to develop the profession to its present state, where restorative and curative ministrations greatly predominate. It is thought by many that the dentistry of the future will be largely preventive and the best minds of science and art were concerned on efforts to consummate this much desired wish and strongly propounded belief.'

\section{REFERENCES}

1. Schewe EF, Black GV. The man of the centuries. Washington University Dental Journal 1950 May. Available at: http:/ /beckerexhibits.wustl.edu/dental/articles/Black_Schewe.html

2. Heyman OH,SwiftEJ, Ritter AV.Sturdevant's art and science of operativedentistry.6thed.Louis,Missouri:ElsevierMosby,2013.

3. Gopikrishna V. Preclinical manual of conservative dentistry. 1st ed. India: Elsevier; 2011.

4. Mc Gehee. A Text Book of Operative Dentistry, 1947.

5. Jagadish S. Conservative class II amalgam cavity preparation. Monograph on recent advances in operative dentistry, 1985.

6. Mount GJ, Hume WR. Preservation and restoration of tooth structure. 1st ed. Mosby International; Tavistock square, London; 1988.

7. Frencken JE, Holmgren CJ. Atraumatic restorative treatment for dental caries. Nijmegan, Netherlands: STI book BV;1999. 